\title{
Prorenin receptor: no role in the RAS?
}

A new study shows that nephron-specific knockout of Atp6ap2 - also known as the prorenin receptor - results in impaired V-ATPase activity and distal renal tubular acidosis in mice, but does not affect the renin-angiotensin system (RAS). "This finding is very important for the RAS field in which Atp6ap2 is still highly studied," says researcher Matias Simons.

Atp6ap2 is a transmembrane protein and accessory subunit of V-ATPase that has been reported to bind renin and prorenin. "Due to the ongoing debate about whether Atp6ap2 really is the long-sought prorenin receptor, we teamed up with the Eladari group to analyse a nephron-specific, inducible Pax8-Cre knockout," explains Simons. "We were mainly interested in post-developmental phenotypes as a previous study found that early deletion of Atp6ap2 causes renal hypodysplasia."

The knockout mice had impaired renal acid-base regulation owing to reduced V-ATPase expression and activity in the intercalated cells of the collecting duct. They were also polyuric and further analysis suggested that this failure to concentrate urine was caused by downregulation of the ion transporters NKCC2 and AQP2 as a result of an autophagic defect in the epithelial cells of the medullary tubules. Renal renin expression, angiotensin II production, sodium handling and blood pressure responses to angiotensin II were similar in knockout and control mice, suggesting that deletion of Atp6ap2 does not affect the intrarenal RAS.

"Our findings demonstrate an important role of Atp6ap2 in V-ATPase activity, both for intracellular events such as autophagy and for plasma membrane events such as acid secretion by intercalated cells," says Simons. "The V-ATPase phenotypes are in line with previous work from our group in Drosophila melanogaster, which does not have a RAS." Although their results do not rule out a role of Atp6ap2 in RAS-independent renin signalling, the researchers conclude that the adverse effects of Atp6ap2 depletion argue against the development of inhibitors of this protein for the treatment of hypertension and kidney disease.

Ellen F. Carney

ORIGINAL ARTICLE Trepiccione, F. et al. Renal Atp6ap2/(Pro) renin receptor is required for normal vacuolar $\mathrm{H}^{+}$-ATPase function but not for the renin-angiotensin system.J. Am. Soc. Nephrol. http://dx.doi.org/10.1681/ASN.2015080915 (2016) 